\title{
TITLE:
}

\section{Quantum Zeno effect and adiabatic change}

$\operatorname{AUTHOR}(S)$ :

Kitano, M.

\section{CITATION:}

Kitano, M.. Quantum Zeno effect and adiabatic change. Physical Review A 1997, 56(2): 1138-1141

ISSUE DATE:

1997-08

URL:

http://hdl.handle.net/2433/152445

RIGHT:

(C) 1997 The American Physical Society 


\title{
Quantum Zeno effect and adiabatic change
}

\author{
M. Kitano* \\ Department of Electronics and Communication, Kyoto University, Kyoto 606-01, Japan
}

(Received 11 September 1996; revised manuscript received 25 March 1997)

\begin{abstract}
We show that the quantum Zeno effect and the adiabatic change have a close connection even though the former means a halt of the dynamics by frequent measurements and the latter is the dynamics by a slowly changing Hamiltonian. We investigate the motion of a spin under stepping magnetic fields in terms of the (inverse) quantum Zeno effect. The relation between this model and the adiabatic change is studied. Another model is used to integrate the two effects into one formulation which is an extension of the proof of the adiabatic theorem. Despite their good parallelism, the asymptotic behaviors of the transition probability with respect to the total period $T$ have different forms; the former effect shows a $T^{-1}$ behavior while the latter scales $T^{-2}$. [S1050-2947(97)00908-6]

PACS number(s): 03.65.Bz, 42.50.-p
\end{abstract}

\section{INTRODUCTION}

The quantum Zeno effect is the suppression or the inhibition of the free dynamics of a system by frequent or continual measurements [1]. The projection associated with measurements effectively destroys the coherence, only through which the transition from one state to another could take place. So the essential ingredient of the Zeno effect is not literal quantum measurement but destruction of coherence, which can be provided by other means such as optical pumping [2], collisions, noisy external fields, and so on. We even can find classical realizations of the Zeno effect; for example, in a system of two coupled pendulums, we can suppress the excitation transfer from one pendulum by giving strong damping to the other pendulum. The damping accompanies no energy losses.

It is very interesting to view the quantum Zeno effect from a rotating frame in which the free dynamics of the system is canceled. In this frame, a series of projections is performed toward slightly different states one after another and the system evolves after these states. This effect was discussed by Aharonov and Vardi [3] and was called the inverse quantum Zeno effect by Altenmüller and Schenzle [4].

This active view of the quantum Zeno effect suggests its applicability to manipulation of quantum systems because we can guide the system as prescribed. In spite of the nonunitarity of evolution, the probability amplitude is almost conserved. We also note that the situation is very similar to that of the adiabatic change of quantum systems in which the state is evolved so as to be an eigenstate of the instantaneous Hamiltonian at each time. The adiabatic change is widely used to control quantum systems.

In this paper, we investigate the relation between the quantum Zeno effect and the adiabatic change. For simplicity, we will consider a system of spin $1 / 2$. First we will present a simple discrete model for the inverse Zeno effect in Sec. II. In Sec. III, we analyze the motion of a spin under stepping magnetic fields. When the number of steps is in-

\footnotetext{
*Electronic address: kitano@kuee.kyoto-u.ac.jp
}

creased, the spin follows the magnetic field like an adiabatic change. Comparing these two models, we present a close analogy between the adibatic change and the inverse Zeno effect.

In Sec. IV we introduce a continuous model with a spin $1 / 2$ under the action of a magnetic field and a coherence relaxation, both of which are slowly moving in time. We examine the long-time asymptotic behavior of the transition probability. The result can be understood as an extension of the adiabatic theorem. In Sec. V, we illustrate numerical solutions of the equations of motion.

\section{ZENO EFFECT IN ROTATING FRAME}

In the usual setting of the quantum Zeno effect, frequent projections to one fixed state halt the system dynamics from that state to other states. Let us look at the phenomenon from a moving frame in which the system dynamics is canceled. In this frame, each projection takes place toward a different state one after another and the system follows it.

Let us consider a spin $\boldsymbol{R}$ for which a series of projections is applied. The axes of projections are defined as

$$
\boldsymbol{u}_{k}=-\sin \frac{k \pi}{n} \boldsymbol{e}_{2}+\cos \frac{k \pi}{n} \boldsymbol{e}_{3},
$$

where $\left(\boldsymbol{e}_{1}, \boldsymbol{e}_{2}, \boldsymbol{e}_{3}\right)$ are the Cartesian unit vectors.

By the projection to $\boldsymbol{u}_{1}$, the initial state $\boldsymbol{R}_{0}=\boldsymbol{e}_{3}$ is transformed to

$$
\boldsymbol{R}_{1}=\left(\boldsymbol{R}_{0} \cdot \boldsymbol{u}_{1}\right) \boldsymbol{u}_{1}=\cos \frac{\pi}{n} \boldsymbol{u}_{1}
$$

After $n$ projections, we have a spin-flipped state

$$
\boldsymbol{R}_{n}=-\cos ^{n} \frac{\pi}{n} \boldsymbol{e}_{3}
$$

The asymtotic behavior of the factor is

$$
\cos ^{n} \frac{\pi}{n}=1-O\left(\frac{1}{n}\right), n \rightarrow \infty .
$$


Thus we can make a spin follow a dense sequence of projections along a presumed path without loss of its length.

For realistic systems each projection requires a finite time $\tau_{k}$ and therefore the total time $T$ for transfer must satisfy the condition

$$
T>\sum_{k} \tau_{k} \sim n \bar{\tau}
$$

where $\bar{\tau}$ is a typical value of $\tau_{k}$. Therefore, $T$ must be increased according as $n$ is increased.

The situation where the direction of spin is made to follow a prescribed path reminds us of the adiabatic change of a spin by a slowly moving magnetic field.

\section{DISCRETE MODEL TOWARDS ADIABATIC CHANGE}

Let us consider the motion of a spin $\boldsymbol{R}(t)$ driven by a magnetic field $\boldsymbol{B}(t)$ stepping $n$ times during the time interval $[0, T]$. The magnetic field $\boldsymbol{B}(t)$ is kept constant at $\boldsymbol{B}_{k}=B_{k} \boldsymbol{u}_{k}$ during the subintervals $((k-1) T / n, k T / n)$ $(k=1, \ldots, n)$, where $\boldsymbol{u}_{k}$ are defined by Eq. (1).

In the first interval the spin does not move if we set the initial condition $\boldsymbol{R}(0)=\boldsymbol{e}_{3}$.

At $t=T / n, \boldsymbol{B}(t)$ is suddenly switched from $\boldsymbol{B}_{0}$ to $\boldsymbol{B}_{1}$ and then the spin starts to precess about $\boldsymbol{B}_{1}$ with the precession angle $\pi / n$ :

$$
\boldsymbol{R}(t)=\boldsymbol{e}_{3} \cos \phi+\boldsymbol{u}_{1} \cos \frac{\pi}{n}(1-\cos \phi)-\boldsymbol{e}_{1} \sin \phi,
$$

where $\phi=\gamma_{\mathrm{g}} B_{1}(t-T / n)$ and $\gamma_{\mathrm{g}}$ is the gyromagnetic ratio.

The next switching occurs at $t=2 T / n$. Here we assume that the timing of switching has an ambiguity much larger than the precession period, $2 \pi / \gamma_{\mathrm{g}} B_{1}$. This assumption is reasonable when the precession is fast enough. We will consider the statistical ensemble average with respect to the switching time. The averaged (mixed) spin state just before the switching can be represented as

$$
\boldsymbol{R}_{1}=\langle\boldsymbol{R}(t)\rangle_{\phi}=\cos \frac{\pi}{n} \boldsymbol{u}_{1} .
$$

After the switching the averaged spin precesses about $\boldsymbol{B}_{2}$ with the precessing angle $\pi / n$.

Repeating the same procedure, we reach the final state

$$
\boldsymbol{R}_{n}=-\cos ^{n} \frac{\pi}{n} \boldsymbol{e}_{3},
$$

where we have taken the $n$-fold (statistically independent) averages with respect to the switching jitters.

The spin has been flipped from $\boldsymbol{e}_{3}$ to $-\boldsymbol{e}_{3}$ but its length is diminished by the factor $\cos ^{n}(\pi / n)$ owing to the average operations. However, this factor scales as $1-O(1 / n)$ for large $n$; therefore, we can make the final state as pure as possible by increasing $n$ in spite of the averaging operations.

The spin transport by a stepping magnetic field is just a realization of the inverse Zeno effect formulated in Sec. II, because the average performed by Eq. (7) corresponds to the von Neumann projection (2). The decoherence induced by projections plays an essential role in the quantum Zeno effect. In the present case, the coherence is destroyed by the statistical average with respect to the switch timing of the magnetic field.

At first sight, in the limit of $n \rightarrow \infty$, the present model seems to be reduced to the adiabatic following to the slow and smooth magnetic field. For adiabatic changes, the loss of probability (or spin length) is proportional to $T^{-2}$ [5]. In the present case, the total time $T$ must be long enough to accommodate all the switching jitters:

$$
T>\sum_{k} \frac{2 \pi}{\gamma_{\mathrm{g}} B_{k}} \sim \frac{2 \pi}{\gamma_{\mathrm{g}} \bar{B}} n
$$

where $\bar{B}$ represents a typical value of $B$. With the help of Eq. (4), it is found that the loss of probability is proportional to $T^{-1}$ rather than $T^{-2}$.

This contradiction comes from the fact that the stepping magnetic field does not satisfy the adiabatic condition even in the limit of $n \rightarrow \infty$. A series of stepping functions can approximate a smooth function, which is assumed in the adiabatic theorem, in its value but not in its derivative. The high-frequency components due to the discontinuities of the derivative promote the transition.

We note, however, that our present model retains the principal feature of the adiabatic process in the sense that the spin follows the moving magnetic field and that the slower the change of field is, the more faithfully the spin follows.

\section{CONTINUOUS MODEL}

In the last section we introduced a discrete model with which the connection between the adiabatic change and the inverse quantum Zeno effect is inspired. In this section we generalize a proof of adiabatic theorem [5] by including a relaxation term which accounts for the Zeno effect.

We consider a spin $1 / 2$ driven by a magnetic field and a relaxation both of which varies in time from $t_{0}$ to $t_{1}$ continuously. We use a normalized time $s=\left(t-t_{0}\right) / T$ with the total period $T=t_{1}-t_{0}$. We will investigate the asymptotic behavior of the system with respect to $T$.

We represent the continuous change of the magnetic fields as $\boldsymbol{B}(s)=\gamma_{\mathrm{g}}^{-1} \Omega(s) \boldsymbol{u}(s)$. We suppose that the unit vector $\boldsymbol{u}(s)$ is a continuous function of $s$ and $d \boldsymbol{u}(s) / d s$ is a piecewise continuous function.

It is assumed that the relaxation axis coincides with the direction $\boldsymbol{u}(s)$ of the magnetic field. The spin components orthogonal to the axis relax with the rate $\Gamma(s)$ while the component along the axis does not relax. This kind of relaxation results in the projection to the axis.

The equation of motion for the $(2 \times 2)$ density matrix $\rho(s)$ can be written as

$$
i \hbar T^{-1} \frac{d \rho}{d s}=\frac{\hbar \Omega(s)}{2}[\rho, \sigma]+i \frac{\hbar \Gamma(s)}{2}(\sigma \rho \sigma-\rho),
$$

where $\sigma=\sigma(s)=\boldsymbol{u}(s) \cdot \boldsymbol{\sigma}$ and $\boldsymbol{\sigma}=\left(\sigma_{1}, \sigma_{2}, \sigma_{3}\right)$ are the Pauli spin matrices.

Here we introduce a Hermitian operator $K(s)$ by which $\sigma(s)$ is evolved, 


$$
i \hbar \frac{d \sigma}{d s}=[K, \sigma]
$$

starting from $\sigma(0)=\sigma_{3}$ at $s=0 . K(s)$ can be expressed as

$$
K(s)=\frac{\hbar \omega(s)}{2} \boldsymbol{v}(s) \cdot \boldsymbol{\sigma},
$$

where $\boldsymbol{u} \times d \boldsymbol{u} / d s=\omega \boldsymbol{v}$. The vector $\omega \boldsymbol{v}$ can be interpreted as the effective field induced by the motion of the reference frame and is piecewise continuous; i.e., it could have discontinuous jumps at finite points.

We define the integrated, evolution operator $A(s)$, which satisfies

$$
i \hbar \frac{d A}{d s}=K A,
$$

with $A(0)=I$. With the unitary transformation $A(s)$, we get the equation of motion for $\widetilde{\rho}(s)=A^{\dagger} \rho A$ in the moving frame,

$$
i \hbar \frac{d \tilde{\rho}}{d s}=[\tilde{\rho}, \widetilde{K}]+\frac{\hbar T \Omega(s)}{2}\left[\tilde{\rho}, \sigma_{3}\right]+\frac{i \hbar T \Gamma(s)}{2}\left(\sigma_{3} \tilde{\rho} \sigma_{3}-\tilde{\rho}\right),
$$

where $\widetilde{K}=A^{\dagger} K A$.

We can write down the equations for the matrix elements of $\widetilde{\rho}, \widetilde{\rho}_{12}$ and $\widetilde{\Delta}=\widetilde{\rho}_{11}-\widetilde{\rho}_{22}$ :

$$
\begin{aligned}
& i \frac{d \tilde{\rho}_{12}}{d s}=-\beta T \tilde{\rho}_{12}+\alpha \widetilde{\Delta}, \\
& i \frac{d \widetilde{\Delta}}{d s}=2 \alpha^{*} \tilde{\rho}_{12}-2 \alpha \tilde{\rho}_{12}^{*},
\end{aligned}
$$

where $\quad \beta=\beta^{\prime}+i \beta^{\prime \prime}=\Omega(s)+i \Gamma(s), \quad \alpha=\alpha^{\prime}+i \alpha^{\prime \prime}, \quad$ and $\widetilde{K}=\hbar\left(\alpha^{\prime} \sigma_{1}-\alpha^{\prime \prime} \sigma_{2}\right)$. Now we see that the real part $\beta^{\prime}$ corresponds to the adiabatic change and the imaginary $\beta^{\prime \prime}$ corresponds to the Zeno effect.

The first equation can be integrated if we assume $\widetilde{\Delta}(s) \sim \widetilde{\Delta}(0)=1:$

$$
\begin{aligned}
\tilde{\rho}_{12}(s) & =-e^{i T \gamma(s)} \int_{0}^{s} i \alpha(\sigma) e^{-i T \gamma(\sigma)} d \sigma \\
& =\frac{\alpha(s)}{T \beta(s)}-\frac{1}{T} e^{i T \gamma(s)} \int_{0}^{s} \frac{d}{d \sigma}\left(\frac{\alpha}{\beta}\right) e^{-i T \gamma(\sigma)} d \sigma,
\end{aligned}
$$

where $\gamma(s)=\gamma^{\prime}+i \gamma^{\prime \prime}=\int_{0}^{s} \beta(\sigma) d \sigma$. It is assumed $\tilde{\rho}_{12}(0)$ $=0$.

Substitution of $\tilde{\rho}_{12}$ into Eq. (16) and its integration yields

$$
\tilde{\rho}_{22}(1)=-\frac{1}{2}(\widetilde{\Delta}(1)-1)=-2 \operatorname{Im} \int_{0}^{1} \alpha^{*} \tilde{\rho}_{12} d s,
$$

which represents the probability of transition. Let us consider the two extreme cases $\beta=i \beta^{\prime \prime}$ (Zeno case) and $\beta=\beta^{\prime}$ (adiabatic case).
For the Zeno case, the first term of Eq. (17) dominates. This approximation is equivalent to neglecting the left-hand side of Eq. (15): $\widetilde{\rho}_{12}=-i\left(\alpha / T \beta^{\prime \prime}\right) \widetilde{\Delta}$. It is interesting to remember that sometimes this procedure is called the adiabatic elimination of variable (in the present case $\rho_{12}$ ) [6]. Equation (18) can be written as

$$
\tilde{\rho}_{22}(1)=\frac{2}{T} \int_{0}^{1} \frac{|\alpha(s)|^{2}}{\beta^{\prime \prime}(s)} d s=O\left(\frac{1}{T}\right) .
$$

For the adiabatic case, the first term of Eq. (17) has no effects on Eq. (18) and therefore the second term has to be estimated. We obtain

$$
\tilde{\rho}_{22}(1)=\frac{2}{T^{2}} \operatorname{Re} \int_{0}^{1} \frac{d}{d s}\left(\frac{\alpha^{*} F}{\beta^{\prime}}\right) e^{i T \gamma^{\prime}} d s=O\left(\frac{1}{T^{2}}\right),
$$

where

$$
F(s)=\int_{0}^{s} \frac{d}{d \sigma}\left(\frac{\alpha}{\beta^{\prime}}\right) e^{-i T \gamma^{\prime}} d \sigma .
$$

Normally, this integration $F$ seems to tend to zero as $T$ increases because of the oscillating factor whose frequency is proportional to $T$. But in some unfavorable cases where $\alpha$ contains high-frequency components, the integration could be independent of $T$. The asymptotic form of the Fourier transform $f(\omega)$ of $\alpha / \beta^{\prime}$, which is a piecewise continuous function of $s$, could scale $\omega^{-1}$ and then the Fourier transform of its derivative behaves as $\omega^{0}$ and $F(s)=O\left(T^{0}\right)$. (Strictly speaking the derivative of $\alpha / \beta^{\prime}$ is not a function but a generalized function because it contains $\delta$ function components.) The same argument can be applied to the evaluation of Eq. (20) and we have found that the transition probability $\tilde{\rho}_{22}(1)$ scales as $O\left(1 / T^{2}\right)$.

We have obtained a unified view which connects the adiabatic change and the quantum Zeno effect by generalizing the formalism for the adiabatic change. We have seen that the transition probability for each case has a different asymptotic behavior for large $T$.

\section{NUMERICAL EXAMPLE: MIXED CASE}

So far we only have investigated the two extreme cases. In order to study a mixed case where the Zeno effect and the adiabatic change coexist, we solve a set of differential equations (15) and (16) with the Runge-Kutta method under the initial condition $\widetilde{\Delta}(0)=1, \widetilde{\rho}_{12}(0)=0$.

We keep the amplitude of the magnetic field $\beta^{\prime}(s)$ and the relaxation rate $\beta^{\prime \prime}(s)$ constant and write

$$
\beta=\frac{1}{2}[(1-k)+i k] \quad(0 \leqslant k \leqslant 1) .
$$

Here the parameter $k$ represents the relative strength between the relaxation rate and the magnetic field; $k=1$ corresponds to the Zeno case and $k=0$ to the adiabatic case. As the motion-induced field, we use the constant form $\alpha(s)=\pi / 2$ which would flip over the spin during the unit time or $\tilde{\rho}_{22}(1)=1$, if $T=0$. 


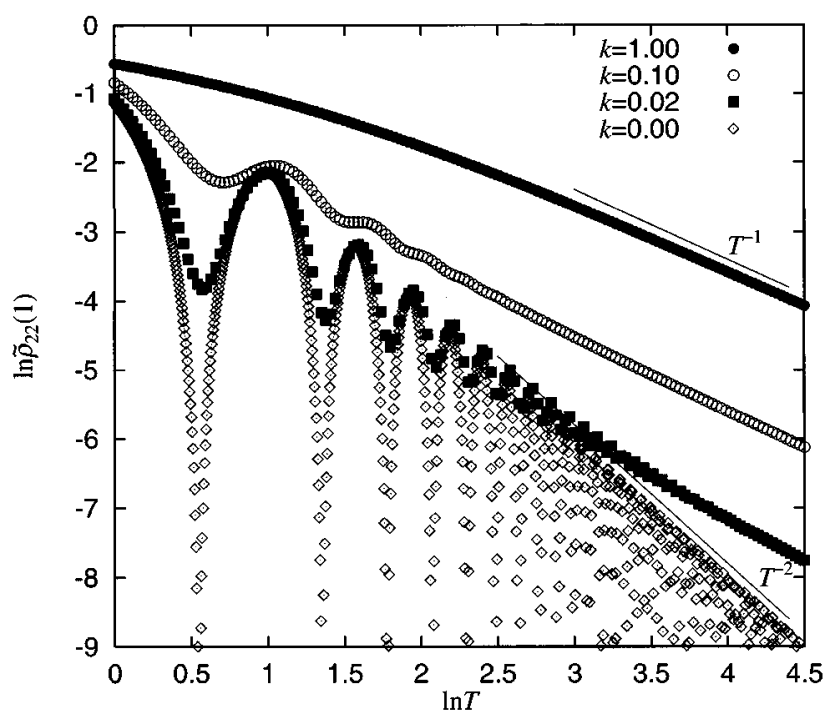

FIG. 1. The probability of spin flipping, $\widetilde{\rho}_{22}(1)$, is plotted as a function of $T$. It scales $T^{-1}$ for Zeno case $(k=1)$ and $T^{-2}$ for adiabatic case $(k=0)$.

The transition (or flipping) probabilities $\tilde{\rho}_{22}(1)$ are (log$\log$ ) plotted as functions of $T$ in Fig. 1. For $k=0, \tilde{\rho}_{22}(1)$ oscillates rapidly, but the peak values scales as $T^{-2}$. For $k=1$, we see the smooth curve which asymptotically tends to $T^{-1}$. In order to see intermediate cases $(0<k<1)$, we plotted the curves for $k=0.1$ and $k=0.01$. In either case, we see the crossover from $T^{-2}$ (adiabatic) to $T^{-1}$ (Zeno) as $T$ increases.

\section{CONCLUSION AND DISCUSSION}

In this paper, we have investigated the relation between the inverse quantum Zeno effect and the adiabatic change.
We have introduced a discrete and a continuous model. In the former model, the spin follows the stepping magnetic field. The phenomenon seemingly resembles to the adiabatic change but should be regarded as an example of the inverse Zeno effect because of its asymptotic behavior. The destruction of coherence, which is essential to the Zeno effect, is caused by the jitter of the stepping magnetic field.

The latter model enabled us to integrate the two effects into one formulation which is an extension of the proof of the adiabatic theorem. In this formulation, the real part of the diagonal elements of the evolution operator corresponds to the magnetic field which accounts for the adiabatic change, while the imaginary part corresponds to the relaxation of the coherence which induces the Zeno effect. Despite the good parallelism, the long-time contributions to the transition probability have different forms; the former shows a $T^{-2}$ behavior while the latter scales $T^{-1}$. We have shown a numerical example where two effects coexist.

Our discrete model belongs to a class of dynamics akin to the adiabatic one. By extending the discussion in Sec. III, it might be possible to understand the proper adiabatic process in terms of the quantum Zeno effects; a slow movement of magnetic field incoherent to the rapid spin precession cannot flip the spin effectively.

We suggested that the inverse quantum Zeno effect can be used to manipulate quantum systems without losses via nonunitary evolutions. We are now carrying out an experiment in order to demonstrate such effects.

\section{ACKNOWLEDGMENTS}

This research was supported in part by the Ministry of Education, Science, Sports, and Culture in Japan, under a Grant-in-Aid for Scientific Research and the VBL Project of Kyoto University.
[1] B. Misra and E. C. G. Sudarshan, J. Math. Phys. (N.Y.) 18, 756 (1977).

[2] W. M. Itano, D. J. Heinzen, J. J. Bollinger, and D. J. Wineland, Phys. Rev. A 41, 2295 (1990).

[3] Y. Aharonov and M. Vardi, Phys. Rev. D 21, 2235 (1980).
[4] T. P. Altenmüller and A. Schenzle, Phys. Rev. A 48, 70 (1993).

[5] A. Messiah, Quantum Mechanics (Wiley, New York, 1966), Chap. 16.

[6] H. Haken, Synergetics (Springer, Berlin, 1978), Chap. 7. 